\title{
Diseño analógico de un estimador de parámetros dependientes de la temperatura de los generadores fotovoltaicos
}

\author{
Analog design of an estimator of temperature \\ dependent parameters of photovoltaic generators
}

Stephanie Barrantes-Pacheco.'

Fecha de recepción: 28 de abril del 2014

Fecha de aprobación: 18 de agosto del 2014

Barrantes-Pacheco, S. Diseño analógico de un estimador de parámetros dependientes de la temperatura de los generadores fotovoltaicos. Tecnología en Marcha. Edición Especial Movilidad Estudiantil 20I4. Pág |4-22 


\section{Palabras claves}

Amplificadores operacionales; energía fotovoltaica; estimador; generadores fotovoltaicos.

\section{Resumen}

Este trabajo consiste en el diseño de un circuito analógico capaz de funcionar como un estimador gradiente para convertidores de energía fotovoltaica. Esto, con el fin de realizar una parte del proceso que se necesita para la conversión de energía fotovoltaica en energía eléctrica. El objetivo principal de este estimador es obtener dos parámetros desconocidos los cuales dependen de la temperatura y la manufactura del panel fotovoltaico que se pretende utilizar. Este diseño de estimador no funciona para todos los generadores fotovoltaicos, pero sí para la mayoría de ellos ya que la propuesta abarca las tecnologías más comunes de generadores que se utilizan actualmente. El documento se divide básicamente en tres partes. Primero se explica un poco la parte teórica y la propuesta en la cual está basado el diseño de este circuito. Luego, con diagramas de bloques se ilustra el funcionamiento que debe tener el circuito propuesto en sus distintas etapas y, por último, se explican las configuraciones analógicas de los circuitos que se encargan de resolver cada uno de los bloques. La unión final de estas configuraciones cumple con el objetivo de obtener los dos parámetros desconocidos.

\section{Keywords}

Estimator; operational amplifiers; photovoltaic energy; photovoltaic generators.

\begin{abstract}
This work involves the design of an analog circuit operable as a gradient estimator for photovoltaic (PV) inverters. This is for a part of the process for the conversion of photovoltaic energy into electrical energy. The main objective of the estimator is to obtain two unknown parameters which depend on the temperature and the PV panel manufacturing intended to be used. This estimator design do not works for all PV generators, but for most of them, because the proposal covers the most common technologies currently used generators. The paper is basically divided in three parts.First, an explication of the theory and the proposal which is based on the design of this circuit is explained. Then, with block diagrams are illustrated the operation that should be in the different stages of the circuit, and finally the analog circuit configurations that are responsible for resolving each of the blocks that are explained. The final combination of these configurations accomplishes the goal of obtaining the two unknown parameters.
\end{abstract}

\section{Introducción}

La energía fotovoltaica es un tema en el que se ha invertido bastante investigación últimamente debido a que se trata de una energía que posee muchas ventajas. Existen distintos tipos de generadores fotovoltaicos; sin importar el tipo de generador que se utilice el proceso para la conversión completa de la energía se divide en dos partes: primero la radiación solar en energía, y luego esta en niveles de corriente y voltaje. Este trabajo se enfoca en la segunda parte del proceso. Para esto requiere tomar en cuenta alguna información relevante en el tema.

\section{Conversión de energía fotovoltaica y generadores fotovoltaicos}

La energía fotovoltaica es un tema en el que se ha invertido bastante investigación últimamente debido a que se trata de una energía que posee muchas ventajas.

Los generadores fotovoltaicos tienen la relación generalizada entre corriente y tensión dada por ( I), donde $\Delta(\cdot)$ y $\varphi(\cdot)$ son funciones que varían según la tecnología del generador de voltaje. $S$ es la incidencia de la radiación solar, $T$ la temperatura del generador y $\rho\left(\rho_{1}, \rho_{2}, \rho_{3}, \ldots, \rho_{n}\right)$ es un conjunto de 
parámetros que dependen del material y la manufactura de los distintos generadores fotovoltaicos.

$$
I_{p v}=\Delta(S, i, T, p)-\phi\left(S, T, v_{p v}, p\right)
$$

En esta ecuación es importante destacar tres características de ella. La primera es que $\varphi(\cdot)$ es estrictamente creciente, la segunda que $i_{p v}\left(v_{p v}\right)$ es biyectiva con respecto a $\boldsymbol{v}$, y por último $\Delta(\cdot)$ es mayor o a lo sumo igual que $\varphi(\cdot)$. Estas características son fundamentales para la ejecución del estimador. Esta ecuación es de suma importancia para poder operar el generador en el punto de máxima potencia.

Algunos de los parámetros $\rho$ que dependen de la temperatura propiamente del generador no se pueden calcular o medir de manera directa por lo que se requiere la estimación de algunos de estos. Por esto, se genera la necesidad de adquirir algún medio que pueda obtener los parámetros deseados. Para obtener estos parámetros se diseñó el estimador propuesto por Carlos Meza y Romeo Ortega.

Se tiene un principal supuesto, se trabaja en condiciones ambientales uniformes. A esto se refiere que se tiene la misma radiación solar y la misma temperatura en cada celda. El estimador podrá ser utilizado para la mayoría de generadores fotovoltaicos ya que la propuesta abarca las tecnologías más comunes de generadores que se utilizan. Cada tecnología varía según el material que se utilice y el proceso de manufactura.

Como se mencionó antes, este trabajo se enfoca en la segunda conversión del proceso, donde se deben estimar el valor desconocido de algunos parámetros. Para esto es que se diseña el estimador, donde se toma la corriente y voltaje provenientes del panel solar como entradas del circuito encargado de estimar los parámetros.

Los valores que se pretenden estimar son de gran importancia para lograr que el generador fotovoltaico opere en su punto de máxima potencia, para así aprovechar al máximo la energía. Por otra parte, estos parámetros son necesarios para algunas estructuras de control que utilizan el modelo eléctrico no lineal de los paneles fotovoltaicos.

Existe un estudio y una propuesta teórica para la creación del estimador que cumple con el objetivo deseado. Este proyecto se tomó como base para continuar con el diseño analógico del estimador descrito.

\section{Estimador de parámetros de generadores fotovoltaicos propuesto}

El estimador propuesto está basado en un modelo simple de generadores fotovoltaicos, no funciona para todas las tecnologías existentes, pero sí para muchas de ellas. El modelo para el cual funciona este estimador está basado en 4 supuestos sobre la ecuación (I). La primera es que $\Delta(\cdot)$ está definida por:

$$
\Delta(\cdot)=p_{1} S
$$

donde $\rho_{1}$ es un parámetros constante positivo, que depende del material y el proceso de manufactura del generador fotovoltaico y $S$ la radiación solar como se dijo anteriormente. La segunda es que $\varphi(\cdot)$ está dada por:

$$
\phi(\cdot)=p_{2}(T) \exp \left(p_{3}(T) v_{p v}\right)
$$

donde $\rho_{2}(T)$ y $\rho_{3}(T)$ son parámetros positivos que dependen también del material y el proceso de manufactura pero, también, de la temperatura del generador fotovoltaico. La tercera es que $\rho_{1}>>\rho_{2}$. Por último, se tiene que el generador fotovoltaico no tiene pérdidas resistivas. Con esto se tiene la ecuación (I) reescrita como se muestra en (2).

$$
i_{p v}\left(v_{p v}\right)=p_{1} S-p_{2} \exp \left(p 3 v_{p v}\right)
$$

Algunos datos importantes sobre distintos parámetros que se involucran en el problema son los siguientes. Los parámetros $\rho_{2}$ y $\rho_{3}$ deben ser estimados debido a que la temperatura del generador no se mide y estos dependen de este factor. La radiación solar y $\rho$, son conocidos, como se explicará más adelante, y por último $v_{p y} y i_{p v}$ se obtienen por medición.

Para la obtención del parámetro $\rho$, se realiza un pequeño experimento con el panel fotovoltaico que se pretende utilizar. Se obtiene la gráfica de radiación solar contra la corriente del panel en corto circuito, con variaciones de la radiación. Con esto se obtiene una recta de la cual se calcula la pendiente y este es el valor del parámetro $\rho_{1}$. Para obtener $v_{p v} y i_{p v}$ se mide la tensión y corriente de la celda o el panel fotovoltaico. 
Para los parámetros $\rho_{2}$ y $\rho_{3}$ se realiza la estimación de este trabajo, debido a que los valores que interesan son los de los parámetros y no la temperatura del generador propiamente. Además, realizar el proceso similar para la obtención de $\rho$, es más complicado y de mayor duración que la estimación de los parámetros.

Meza y Ortega señalan en su documento (2) como:

$$
y=\theta^{T} \Phi
$$

donde se tiene que:

$$
\begin{gathered}
\Phi=\left[v_{p v} 1\right]_{T} \\
y=\ln \left(S p_{1}-i_{p v}\right) \\
\theta=\left[\theta_{1} \theta_{2}\right]_{T}
\end{gathered}
$$

y $\theta_{1}$ y $\theta_{2}$ representan los parámetros que se quieren estimar,

$$
\begin{gathered}
\theta_{1}=p_{3} \\
\theta_{1}=\ln \left(p_{2}\right)
\end{gathered}
$$

También definen $\hat{\boldsymbol{\theta}}=\left[\hat{\boldsymbol{\theta}}_{1} \hat{\boldsymbol{\theta}}_{2}\right]^{T}$ como los valores estimados de $\theta$, y con esto definen el estimador como se muestra en (4).

$$
\hat{\theta}=\Gamma \Phi(y-\Phi T \hat{\theta})
$$

Se tiene que $\Gamma$ es una matriz positiva definida por:

$$
\Gamma=\left[\begin{array}{ll}
\gamma_{11} & \gamma_{12} \\
\gamma_{21} & \gamma_{22}
\end{array}\right]
$$

donde cada uno de estos factores se elige de manera adecuada para afinar las dinámicas del estimador.

\section{Propuesta de circuito para estimar los parámetros deseados}

Para la estimación de los parámetros del generador fotovoltaico se propone un circuito analógico conformado por amplificadores operacionales, resistencias, capacitores, diodos y de ser necesario algún circuito integrado que se encargue de alguna operación en específico.

De la ecuación (4), denominando $v_{p v}=w$ se obtienen (5) y (6), las cuales representan la ecuación principal del estimador para obtener los parámetros deseados.

$$
\begin{aligned}
& \dot{\hat{\theta}}_{1}=\left(\gamma_{11} w+\gamma_{12}\right)(\gamma-\hat{\gamma}) \\
& \dot{\hat{\theta}}_{2}=\left(\gamma_{21} w+\gamma_{22}\right)(\gamma-\hat{\gamma})
\end{aligned}
$$

donde

$$
\hat{\gamma}=\hat{\theta}_{1} w+\hat{\theta}_{2}
$$

Las ecuaciones (5) y (6) se pueden reescribir como se muestra en (7) y (8) respectivamente.

$\dot{\hat{\theta}}_{1}=\gamma_{11}\left(w y-w^{2} \hat{\theta}_{1}-w \hat{\theta}_{2}\right)+\gamma_{12}\left(y-w \hat{\theta}_{1}-\hat{\theta}_{2}\right)$
$\dot{\hat{\theta}}_{2}=\gamma_{21}\left(w y-w^{2} \hat{\theta}_{1}-w \hat{\theta}_{2}\right)+\gamma_{12}\left(y-w \hat{\theta}_{1}-\hat{\theta}_{2}\right)$

Para resolver estas ecuaciones, se propone un circuito analógico que se encargue de obtener los valores deseados. El circuito tiene como entradas $w, y, \gamma_{\|}$, $\gamma_{12}, \gamma_{21}$, y $\gamma_{22}$. Las dos primeras son señales medidas u obtenidas directamente a través de mediciones y el resto son definidas por el diseñador. Las salidas de este circuito corresponden a $\dot{\hat{\theta}}_{1}$ y $\dot{\hat{\theta}}_{2}$ como se puede observar en la figural. Este primer diagrama se divide en distintos bloques encargados de resolver cada una de las partes de la ecuación que se mostrará más adelante.

En las ecuaciones (7) y (8) se tienen algunas multiplicaciones de distintas señales las cuales se redefinen de la siguiente manera para su utilización posteriormente:

$$
\begin{gathered}
p_{1}=w y ; \\
p_{2}=w^{2} \hat{\theta}_{1} ; \\
p_{3}=w \hat{\theta}_{2} ; \\
p_{4}=w \hat{\theta}_{1} ;
\end{gathered}
$$

\section{Diagramas de bloques para el estimador}

El bloque que se muestra en la figural contiene internamente distintas funciones. Estas funciones están definidas por los bloques que se muestra en la figura 2, donde cada uno de estos tiene una función específica. Los bloques Ec.(5) y Ec.(6) se requieren para resolver cada una de las ecuaciones de manera individual. En este diagrama se observa que los dos bloques se encargan de obtener las ecuaciones (5) y (6), donde la salida de estas es integrada para obtener las salidas correspondientes $\dot{\hat{\theta}}_{1}$ y $\dot{\hat{\theta}}_{2}$. 


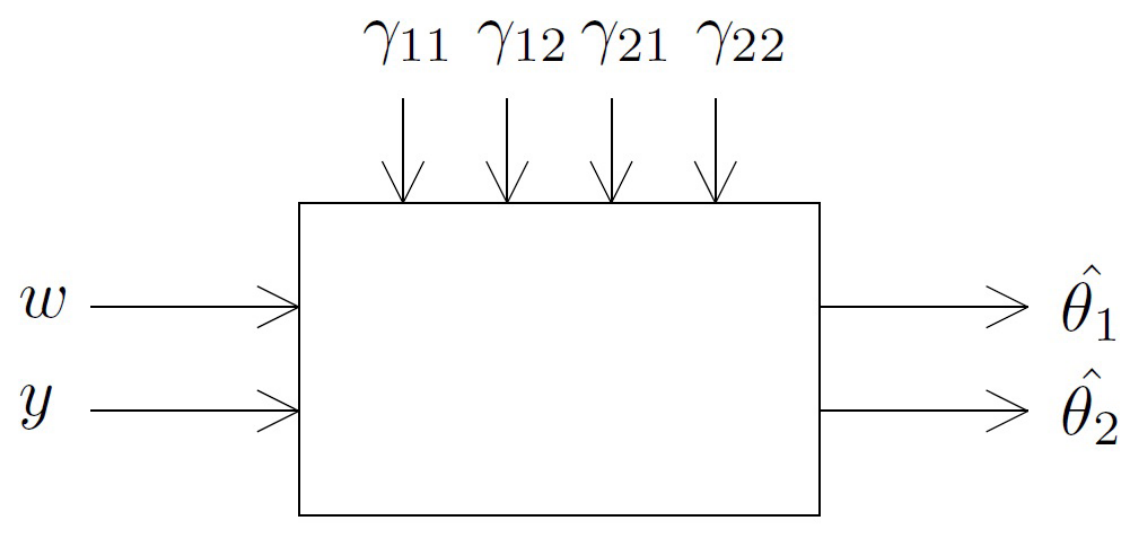

Figura I: Diagrama general para resolver las ecuaciones (5) y (6).

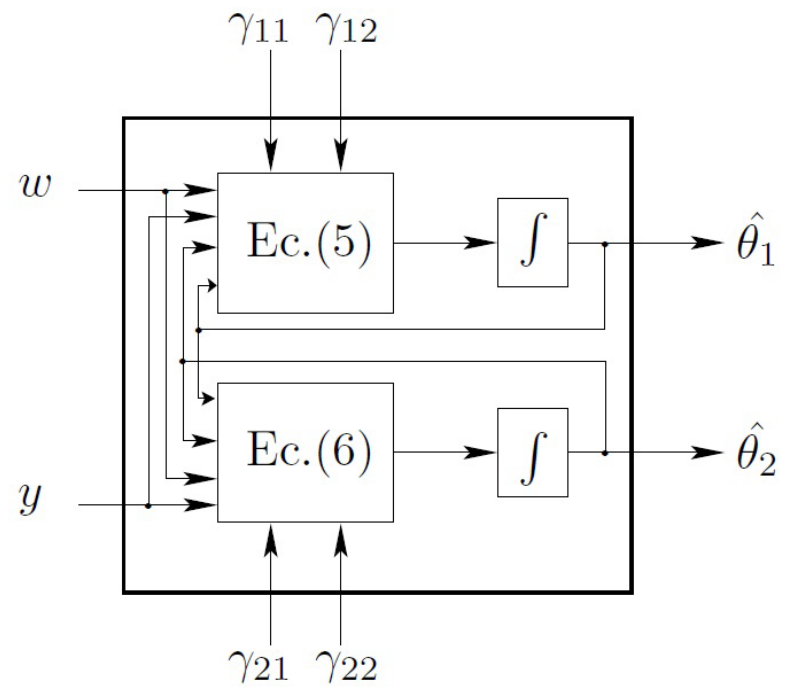

Figura 2: División de bloques internos del diagrama general.

Cada uno de los bloques encargados de resolver las ecuaciones está conformado por una configuración como la del diagrama de la figura 3. En esta figura los bloques de la izquierda se encargan cada uno de obtener, $p_{1}, p_{2}, p_{3}$ y $p_{4}$. Luego, las señales $p_{1}, p_{2}, p_{3}$ se suman para ser escaladas con una ganancia de $\gamma_{x 1}$, donde $\boldsymbol{x}$ puede tomar el valor de 1 o 2, según sea la ecuación que se está resolviendo para escalar con el valor correspondiente. De la misma manera sucede con las señales $p_{4^{\prime}}$ y y $\dot{\hat{\boldsymbol{\theta}}}_{2}$, que se suman para amplificarlas con $\gamma_{\times 2}$. Estas dos salidas amplificadas son sumadas para obtener $\dot{\hat{\theta}}_{1} \circ \dot{\hat{\theta}}_{2}$ según sea el caso, y proceder a integrarlas para obtener $\dot{\hat{\theta}}_{1}$ y $\dot{\hat{\theta}}_{2}$ y respectivamente.

\section{Obtención de señales del panel fotovoltaico}

Para realizar la estimación deseada se necesitan dos señales provenientes del panel solar que son $i_{p v} y v_{p v}$. Para obtener estas señales se requiere utilizar un circuito de medición como el que se muestra en la figura 4. La tensión $v_{p v}$ es la señal medida directamente entre las terminales del panel y la corriente $i_{p v}$ se obtiene dividiendo la tensión de la resistencia $R_{\text {, }}$ entre el valor de esta. Las resistencias $R m$ y $R$, deben ser de valores bajos para que no afecte significativamente el valor de la tensión del panel.

Las señales que se requieren para el funcionamiento del estimador son las denominadas y y $w$. Donde como se definió anteriormente $y=\ln \left(S p_{1}-i_{p v}\right)$ y $w=v_{p v^{\prime}}$. Para obtener $w$ se utiliza la señal medida directamente de las terminales del panel y para y se debe agregar algunas configuraciones con amplificadores operacionales que resuelven esta ecuación.

Obtención de la señal y

Para obtener la señal $y$, primero se debe tener el valor de $i_{p v}$ y de $S \rho_{l}$, este último se obtiene como se explicó antes. Luego, con estos valores se debe realizar una configuración para restar. Además de esto se crea otra configuración para obtener el logaritmo natural y con esto se obtiene $y$. Esta etapa de obtención de y se observa en la figura 5.

La configuración para obtener el logaritmo natural de una entrada específica se muestra en la figura 6. Acá se seleccionan los valores adecuados de resistencias tomando en cuenta la propiedad de la corriente en el diodo de un logaritmo natural. 


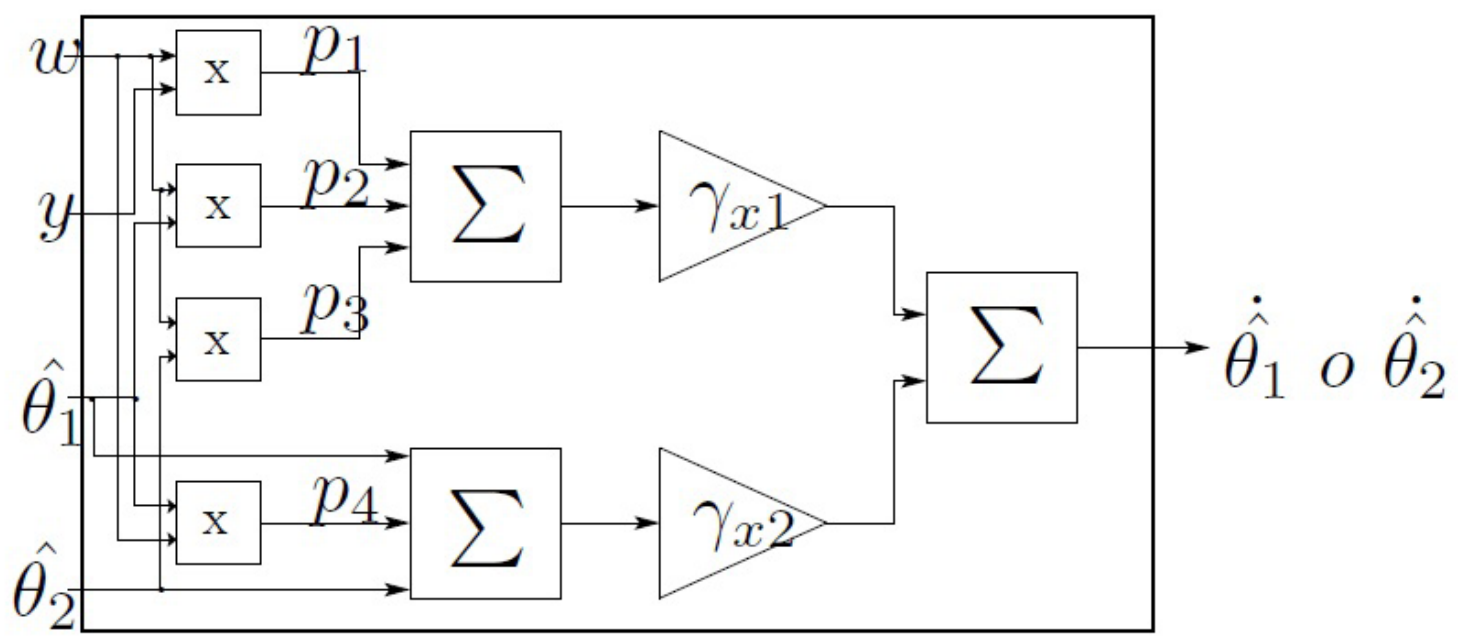

Figura 3: Diagrama de bloques para resolver cada ecuación.

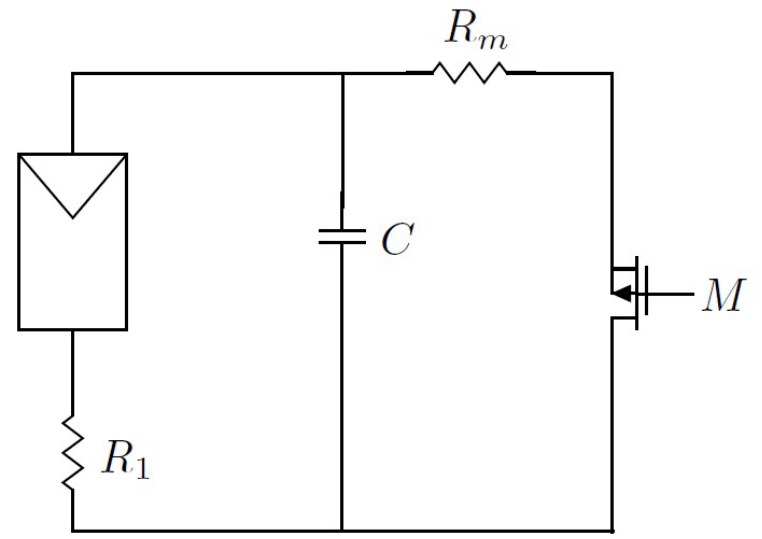

Figura 4: Circuito de medición de $i_{p v} y v_{p v}$

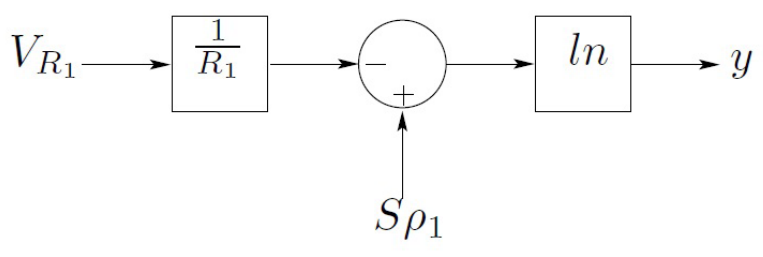

Figura 5: Diagrama de bloques para obtención de $y$.

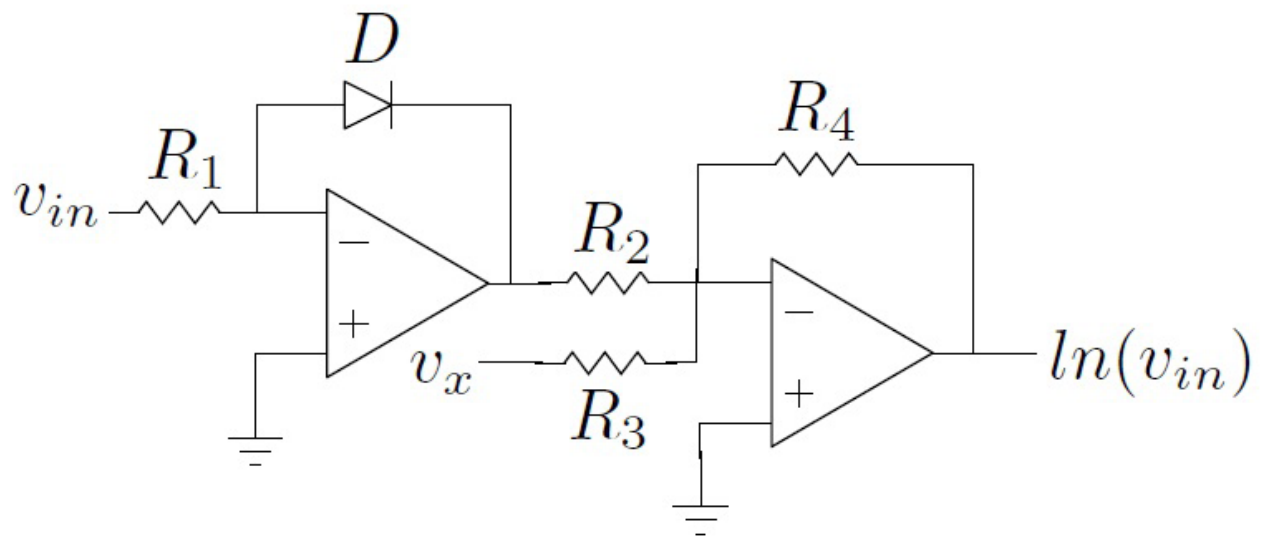

Figura 6: Circuito para obtener el logaritmo natural. 


\section{Diseño de configuraciones analógicas para el circuito estimador de parámetros}

En esta sección se explicarán las configuraciones propuestas para resolver cada etapa de las ecuaciones (5) y (6).

\section{Multiplicación de señales}

Lo primero que se debe resolver para obtener los resultados correctos de las ecuaciones (5) y (6) son las multiplicaciones para obtener $p_{1}, p_{2}, p_{3}$ y $p_{4}$. Cada una de estas se puede resolver utilizando uno o más circuitos de multiplicación, según sea el caso, con el diseño que se muestra en la figura 7. Este circuito se encarga de realizar la multiplicación entre $s_{1}$ y $s_{2}$ por medio del logaritmo, sus entradas son $s_{1}$ y $s_{2} y$ la salida correspondiente es $s_{1} s_{2}$. Para que este circuito funcione de manera correcta todos los diodos deben ser iguales, y lo mismo debe suceder con las resistencias. Con esto, al aplicar el logaritmo natural al inicio y el antilogaritmo al final se cancelan los parámetros del diodo por ser iguales y se obtiene la multiplicación de señales deseada. Para obtener $p_{l}$, $p_{3}$ y $p_{4}$ se utiliza una configuración de este circuito para cada uno, y para $p_{2}$ también es una configuración pero una de sus entradas será $p_{4}$; con esto se evita tener que utilizar dos etapas de multiplicación para la obtención de $p_{2}$.
Suma y resta de señales

Luego de obtener la multiplicación de las distintas señales estas se deben sumar entre sío con alguna de las entradas provenientes del panel. Para realizar estas sumas se utiliza la configuración que se muestra en la figura 8. Donde la salida de este circuito es la suma de las entradas invertida, esto si se cumple que $R_{1}=R_{2}=R_{3}=R_{4}$.

Este circuito, también, se utiliza para restar algunas señales ya que al invertir la señal que se desea restar y colocarla en una de las entradas de este circuito estas se restaran, siempre obteniendo el resultado invertido.

\section{Ganancias variables}

Una vez realizada la suma de las señales provenientes de las multiplicaciones sigue la etapa de las ganancias. Para obtener las ganancias deseadas $\gamma_{11}$, $\gamma_{12}, \gamma_{21}, y \gamma_{22}$, se utilizan amplificadores operacionales, con la configuración de amplificación no inversora mostrada en la figura 9 ajustando sus ganancias con un potenciómetro en $R_{2}$. La ecuación de este circuito está dada por:

$$
\frac{v_{o}}{v_{i}}=\frac{R_{1}+R_{2}}{R_{1}}
$$

$\operatorname{con} R_{3}=R_{1} / / R_{2}$

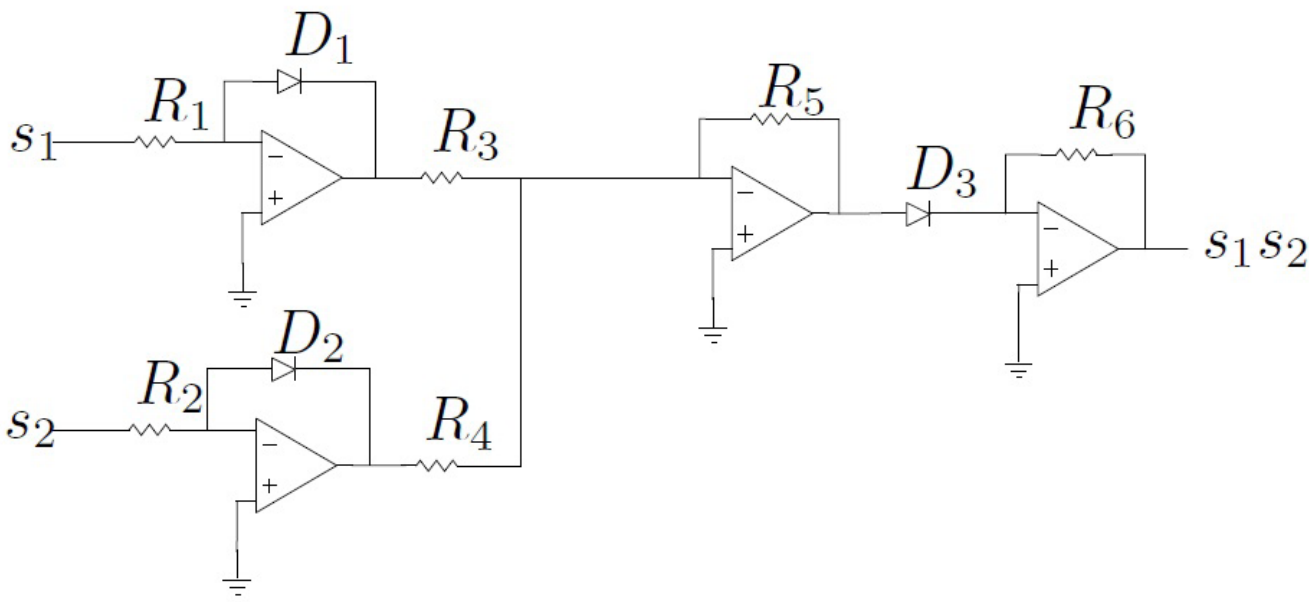

Figura 7: Circuito de multiplicación con amplificadores operacionales. 


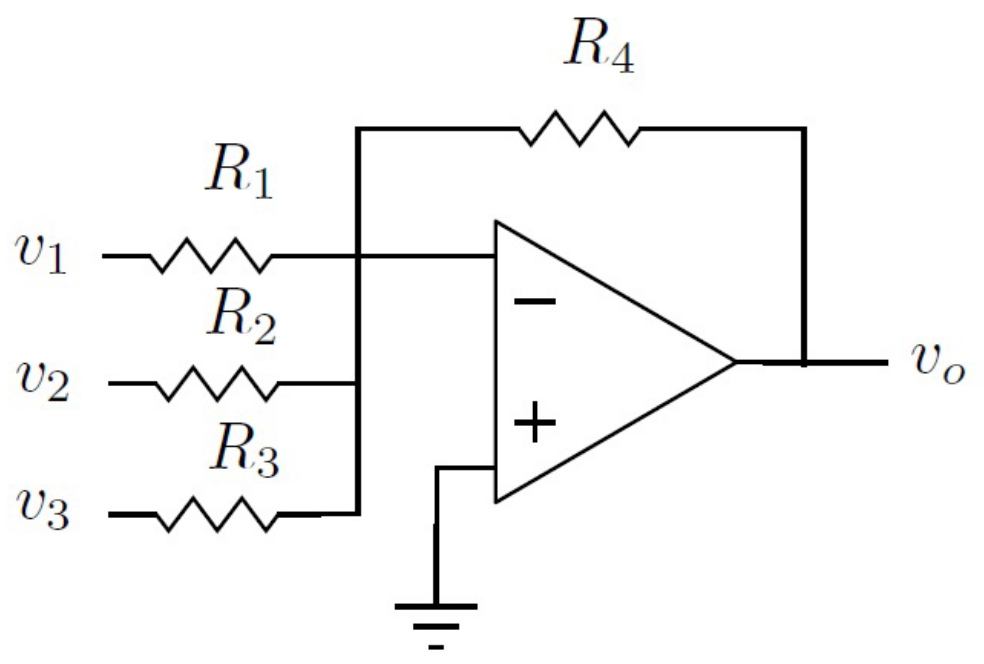

Figura 8: Configuración de sumador inversor.

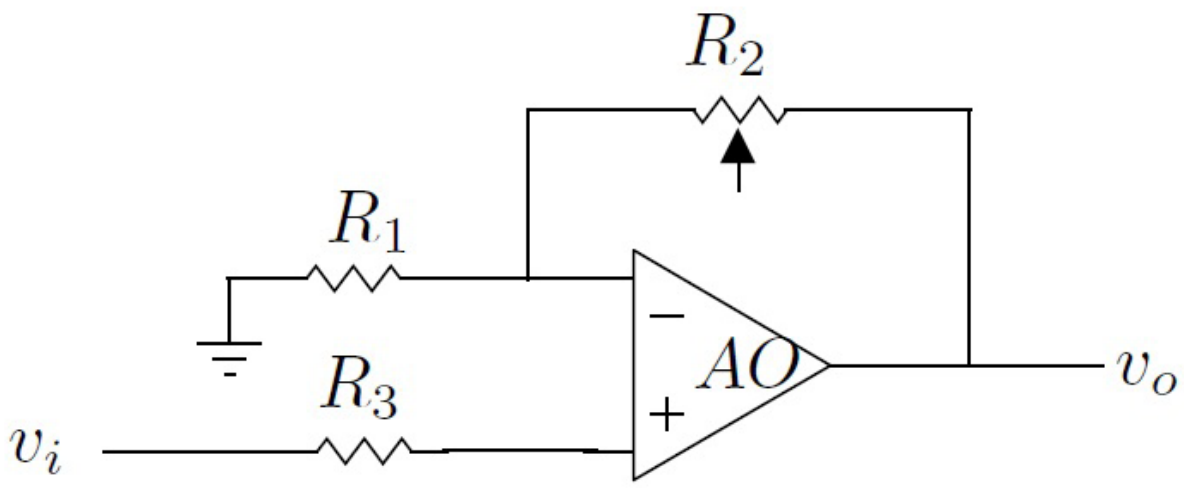

Figura 9: Circuito amplificador no inversor.

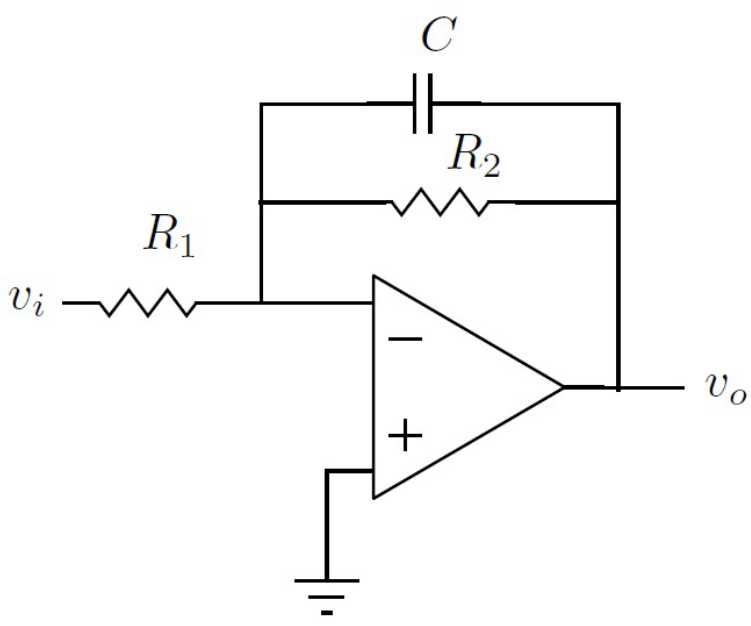

Figura 10: Configuración de circuito integrador
Con las salidas obtenidas de cada bloque de las ganancias se procede a realizar la suma para obtener $\dot{\hat{\theta}}_{1}$ y $\dot{\hat{\theta}}_{2}$, con la configuración de la Fig.8.

Integración de la señal obtenida

Para finalizar la obtención de $\dot{\hat{\boldsymbol{\theta}}}_{1}$ y $\dot{\hat{\theta}}_{2}$ y se deben integrar las señales obtenidas en el paso anterior, y $\dot{\hat{\theta}}_{1}$ y $\dot{\hat{\theta}}_{2}$. Para esto, se deben utilizar dos configuraciones como las de figural0. Esta configuración se encarga de obtener la integral de la entrada con una ganancia que depende de los valores de las resistencias y la capacitancia. Para esto, se deben seleccionar los valores adecuados de estos componentes para que no afecten el resultado que se desea obtener. 


\section{Conclusión}

Con las distintas configuraciones de amplificadores operacionales explicadas en este documento se puede crear el circuito analógico capaz de estimar los valores de los parámetros deseados. Además de estas configuraciones, se debe tomar en cuenta posibles etapas de ganancia que puede que sean necesarias para amplificar o atenuar las señales o algún filtro para obtener una señal sin interferencias exteriores.

El siguiente paso por realizar es la implementación del diseño propuesto en este documento. Para esto, se debe tomar en cuenta el panel con el que se trabajará para definir los parámetros correspondientes de manera correcta.

\section{Referencias}

Fernández, A. (2006) Circuitos con amplificadores operacionales, Universidad de Huelva, España.

Meza, C. and Ortega, R. (Octubre, 20I3). "Control and estimation scheme for PV central inverters", en Information, Communication and Automation Technologies (ICAT), 2013 XXIV International Symposium.

Meza, C. and Ortega, R. (Julio, 2013). "On-line estimation of the temperature dependent parameters of photovoltaic generators", en I I th IFAC International Workshop on Adaptation and Learning in Control and Signal Processing.

Slotine, J. and Li, W. (199I) "Applied nonlinear control" Massachusetts Institute of Technology.USA: Prentice-Hall International. 